\title{
Detection of Legionella pneumophila antigen in urine samples by the BinaxNOW immunochromatographic assay and comparison with both Binax Legionella Urinary Enzyme Immunoassay (EIA) and Biotest Legionella Urin Antigen EIA
}

\author{
JÜRGEN H. HELBIG, SØREN A. ULDUM*, P. CHRISTIAN LÜCK and TIMOTHY G. HARRISON $\dagger$ \\ Medical Microbiology and Hygiene Institute, Technical University Dresden, Germany, * Department of \\ Respiratory Infections, Meningitis and STIs, Statens Serum Institut, Copenhagen, Denmark and †Respiratory
} and Systemic Infection Laboratory, PHLS Central Public Health Laboratory, London

\begin{abstract}
The new BinaxNOW Immunochromatographic (ICT) Assay for the detection of Legionella pneumophila antigens was used to test 535 urine specimens from patients with and without Legionnaires' disease. The specificity, calculated by testing 112 samples from patients with pneumonia of aetiologies other than Legionella infection, and 167 urine specimens from urinary tract infections, was found to be $97.1 \%$ if the manufacturer's guidelines were followed. However, it was determined that the 'false positive' results characterised by very weak bands could be discounted by reexamination of the results at $60 \mathrm{~min}$, yielding a specificity of $100 \%$. With this minor modification of the procedure applied to examination of urine samples from 117 patients with legionellosis confirmed by isolation of $L$. pneumophila and 70 patients who had seroconverted to $L$. pneumophila serogroup 1 , sensitivity was calculated to be $79.7 \%$. In comparison, the sensitivities of the Binax Urinary Antigen Enzyme Immunoassay (EIA) and Biotest Urin Antigen EIA were estimated to be 79.1 and $83.4 \%$, respectively. Eleven cases $(5.9 \%)$ were positive by BinaxNOW assay but negative by Binax or Biotest EIA, or both. The sensitivities of all assays increased to $c .94 \%$ if only diagnosis of cases confirmed by isolation of serogroup $1 \mathrm{~L}$. pneumophila was considered, although the sensitivity for infections caused by $L$. pneumophila serogroup 1 monoclonal antibody (MAb) subgroup Bellingham was significantly lower than for other MAb subgroups. The Biotest EIA recognised $10(45 \%)$ of the 22 cases not caused by L. pneumophila serogroup 1, whereas the two Binax kits detected only three each. The ICT assay BinaxNOW can be recommended as a rapid specific test for the diagnosis of Legionnaires' diseases caused by $L$. pneumophila serogroup 1 , although very weak bands should be interpreted cautiously.
\end{abstract}

\section{Introduction}

The outcome of patients with Legionnaires' disease depends on the speed with which a diagnosis can be established and the initiation of appropriate therapy $[1,2]$. Urinary antigen detection for the diagnosis of Legionnaires' disease has several advantages compared

Received 14 April 2000; revised version received 6 Nov. 2000; accepted 28 Nov. 2000.

Corresponding author: Dr J.H. Helbig (e-mail: Juergen. Helbig@mailbox.tu-dresden.de). with other methods. Culture of legionellae from respiratory specimens requires special media and is relatively slow (3-10 days). Moreover, many patients who develop severe pneumonia are treated initially with a broad-spectrum antibiotic regimen that may reduce the sensitivity of culture [3]. Diagnosis by estimation of serum antibody levels is widely used, but does not often allow the diagnosis to be established during the acute illness. Direct fluorescent antibody staining of respiratory specimens is a very rapid method but the sensitivity is poor $[4,5]$. Because of the disadvantages of the above methods as well as the 
ease with which urine samples may be obtained, many attempts have been made to develop urinary antigen assays with high specificity and sensitivity. Because of the high sensitivity required, radio-immunoassay was often the method of choice [6-8]. However, more recently two enzyme immunoassays (Binax Urinary Antigen EIA and Biotest Urin Antigen EIA) have become available commercially. The widespread availability of these non-radioactive assays is one of the major reasons for increasing numbers of Legionnaires' disease cases being diagnosed by urinary antigen detection. As a consequence, Plouffe et al. [9] proposed that the case definition for definitive Legionnaires' disease should be expanded to include positive urinary assays. Similarly, in 1998, the European surveillance scheme for travel-associated Legionnaires' disease, organised by the European Working Group on Legionella Infections, included the detection of specific Legionella antigen in urine by validated reagents within its definition of a confirmed case of legionellosis.

Recently, a rapid immunochromatographic (ICT) assay (BinaxNOW Legionella Antigen) has been developed for the detection of $L$. pneumophila serogroup 1 antigen in urine samples. The two technical advantages of ICTs over conventional EIAs are the ease with which they can be performed (there is no need for expensive laboratory equipment) and the rapidity with which a diagnosis can be established (15 versus $90 \mathrm{~min})$. The specificity of the BinaxNOW kit was evaluated in this study by testing urine specimens from patients with pneumonia of proven aetiologies other than Legionella infection and from patients with urinary tract infections. The sensitivity of the BinaxNOW was calculated by testing specimens from 187 cases of legionellosis and was compared with the sensitivities of the Binax and Biotest EIAs. Because the majority of the culture-proven cases belong to certain monoclonal subtypes of L. pneumophila serogroup 1, the sensitivity was further examined in vitro with Legionella antigens prepared from strains of three $L$. pneumophila serogroup 1 monoclonal subtypes and two other serogroups.

\section{Materials and methods}

\section{Legionella antigen preparations}

For the theoretical sensitivity studies, simulated urine samples were prepared with strains of L. pneumophila serogroup 1 (strains Philadelphia 1, ATCC 33152; OLDA ATCC 43109; Bellingham, ATCC 43111), serogroup 3 (Bloomington 1, ATCC 33155) and serogroup 6 (Chicago 2, ATCC 33215). Bacteria were grown on buffered charcoal-yeast extract agar supplemented with $\alpha$-ketoglutarate 1\% (BCYE- $\alpha$ ) for $48 \mathrm{~h}$ at $37^{\circ} \mathrm{C}$ in air with $\mathrm{CO}_{2} 2.5 \%$. Organisms were harvested from the plates with a glass rod and suspended in phosphate-buffered saline (PBS). The concentrations of the bacteria were measured by nephelometry with the
McFarland standard as a reference and were adjusted to $1 \times 10^{8} / \mathrm{ml}$. The suspension was then ultrasonicated for six bursts of $30 \mathrm{~s}$ each, separated by 30-s cooling period.

\section{Origin of urine samples}

A total of 535 urine samples from six groups of patients was tested. Group A consisted of 95 specimens from patients with Legionnaires' disease confirmed by isolation of L. pneumophila serogroup 1. Group B consisted of specimens from 22 culture-proven cases, in which L. pneumophila serogroup 2 (one patient), serogroup 3 (12), serogroup 4 (1), serogroup 5 (1), serogroup 6 (5), serogroup 10 (1) and one untypable strain (not serogroups 1-15) had been isolated. Group $\mathrm{C}$ consisted of specimens from 70 cases confirmed by a four-fold or greater rise in specific antibody titre against L. pneumophila serogroup 1 in the indirect immunofluorescent antibody test. Group D comprised 69 urine samples that were Legionella antigen-positive by the Binax Legionella Urinary Enzyme Immunoassay (EIA) or Biotest Legionella Urin Antigen EIA, or both. In groups A-D the first urine sample had been collected from day 1 to day 33 after the onset of symptoms. For controls, group E comprised 112 urine specimens from patients with pneumonia of proven aetiologies other than Legionella infection and group F comprised 167 urine samples from patients with urinary tract infection. Specimens in group $\mathrm{E}$ were obtained from patients diagnosed by culture or antigen detection or serology or PCR (or a combination of these methods) as Streptococcus pneumoniae (56 patients), Mycoplasma pneumoniae (36), Coxiella burnettii (2), Chlamydia spp. (11), respiratory syncytial virus (3) and influenza A/B virus (4) infection. The urinary specimens in group $\mathrm{F}$ yielded $>10^{5}$ bacteria or fungi/ml by culture and which were identified as Acinetobacter calcoaceticus (1 case), A. johnsonii (1), A. Iwoffii (1), Candida albicans (1), Citrobacter braakii (1), Cit. freundii (8), Enterobacter aerogenes (3), Ent. cloacae (2), Enterococcus faecalis (20), Escherichia coli (32), Klebsiella oxytoca (9), K. ozaenae (1), K. pneumoniae (18), Morganella morganii (3), Proteus mirabilis (20), Proteus spp. (2), Prot. vulgaris (1), Providencia rettgeri (1), Pseudomonas aeruginosa (26), Ps. alcaligenes (1), Pseudomonas spp. (2), Salmonella typhimurium (1), Serratia marcescens (1), Staphylococcus aureus (3), Stenotrophomonas maltophilia (1) and Streptococcus spp. (3) by conventional microbiological methods.

\section{Legionella urinary antigen detection kits}

Legionella antigen preparations and urine samples were tested simultaneously in the BinaxNOW Legionella Antigen immunochromatographic (ICT) assay (Binax), Binax Legionella Urinary Enzyme Immunoassay (EIA) (Binax) and Biotest Legionella Urin Antigen EIA (Biotest). The tests were performed and the results 
were calculated according to the manufacturers' instructions with one exception. For the BinaxNOW assay, as recommended by the manufacturers, results were read in the 'window' after $15 \mathrm{~min}$. Bands that appeared very weak were also read after $60 \mathrm{~min}$. This allowed the significance of bands that were only very weakly visible after $15 \mathrm{~min}$ to be re-assessed $45 \mathrm{~min}$ later. The specificity of BinaxNOW was calculated according to the manufacturer's recommendations and compared with results obtained with this modification.

\section{Other methods for diagnosis of legionellosis}

L. pneumophila from respiratory specimens were isolated on BCYE- $\alpha$ agar [4]. Antibodies against $L$. pneumophila in patients' sera were determined by the indirect immunofluorescence antibody test with $L$. pneumophila serogroup 1 antigen [10].

\section{Serogrouping and subgrouping of Legionella isolates}

Clinical isolates were characterised as being $L$. pneumophila on the basis of their reactivity with a species-specific FITC-labelled monoclonal antibody (MAb) (Fresenius, Oberursel, Germany). L. pneumophila was serogrouped with MAbs and polyclonal antibodies as described previously [11]. For the division of $L$. pneumophila serogroup 1 isolates into monoclonal subgroups, MAbs 8/5, 3/1, 8/4, 20/1 and $10 / 6$ [11, 12], or MAb 2, MAb 3, W32 and 33G2 [13], or combinations of these, were used. With these MAbs, the isolates were divided into the subgroups Philadelphia, Allentown, France, Benidorm, Knoxville, OLDA/ Oxford and Bellingham according to the international standard scheme developed by Joly et al. [13].

\section{Statistical analysis}

The $p$ values for differences in the rate of positive results were derived from $\chi^{2} 2 \times 2$ contingency tables.

\section{Results}

\section{Incubation time and specificity of the BinaxNOW assay}

To estimate the specificity of the BinaxNOW assay, urine samples from 112 patients with pneumonia caused by organisms other than legionellae (group E) and 167 urine samples from patients with urinary tract infections (cfu $>10^{5} / \mathrm{ml}$; group F), mostly (94\%) caused by gram-negative bacteria, were tested. Five samples from patients with urinary tract infections caused by E. coli (four samples) or Staph. aureus (one sample) and three urine specimens from patients with pneumonia caused by Str. pneumoniae gave very weakly positive bands after a reaction time of $15 \mathrm{~min}$. Heat treatment of the samples did not influence these results. Both the Binax EIA and Biotest EIA gave negative results with these samples. Nevertheless, the possibility of a polymicrobial pneumonia with Str. pneumoniae and L. pneumophila cannot be completely ruled out. Unfortunately, there were not enough volumes of urine samples from pneumonia patients for concentrating and re-testing the specimens. According to the manufacturer's guidelines, these samples should be considered positive. Consequently, the specificity was calculated to be $97.1 \%$. However, on re-assessment $45 \mathrm{~min}$ later, no increase in intensity was noted for these bands. All other control samples were completely negative after $15 \mathrm{~min}$ and $60 \mathrm{~min}$. Therefore, the manufacturer's procedure was modified by discounting very weak results which did not intensify on prolonged incubation. Thus, with these revised criteria, the specificity of BinaxNOW was calculated to be $100 \%$. Five cases of culture-proven legionellosis also produced bands that were only just visible by eye after $15 \mathrm{~min}$, but their intensity had increased significantly $45 \mathrm{~min}$ later. Therefore, all sensitivities calculated in this study were based on repeated reading of very weak bands after $45 \mathrm{~min}$.

\section{Sensitivity for Legionella antigen preparations}

At present there is no procedure for preparation and quantification of soluble Legionella antigens which are passed in the urine. To compare the sensitivities of the urinary antigen assays, agar-grown legionellae were ultrasonicated and used as a standard antigen preparation. The sonication step resulted in better accessibility of the epitopes to antibodies and, thus, in an increase of reactivity in all three assays when compared with intact bacterial cells (data not shown). Based on the estimated bacterial cell number before ultrasonication, the lower limit of detection was determined for antigen preparations of three L. pneumophila serogroup 1 strains of different monoclonal subtypes (Philadelphia, OLDA and Bellingham) and the type strains of L. pneumophila serogroup 3 and serogroup 6 (Fig. 1). The lowest limit of detection for these simulated samples was $3 \times 10^{4}$ sonicated bacteria/ml for the L. pneumophila Philadelphia antigen in all three assays. For strain OLDA, the sensitivity of the two Binax assays was slightly but insignificantly lower, whereas the Biotest EIA had a limit of detection of $6 \times 10^{5}$ cells $/ \mathrm{ml}$. All three assays could recognise L. pneumophila serogroup 3 and 6 antigens only at concentrations of $\geqslant 5 \times$ $10^{6} / \mathrm{ml}$. Heat treatment $\left(95^{\circ} \mathrm{C}, 10 \mathrm{~min}\right)$ of these prepared antigens had no significant influence on the lower detection limit of the assays.

\section{Detection of Legionella antigens in urine samples from patients with culture-proven legionellosis}

Of 117 culture-proven cases, 95 patients $(81.2 \%)$ had legionellosis caused by L. pneumophila serogroup 1 . In this group (group A), the highest sensitivity was found for the BinaxNOW assay (95.8\%); the sensitivities of 


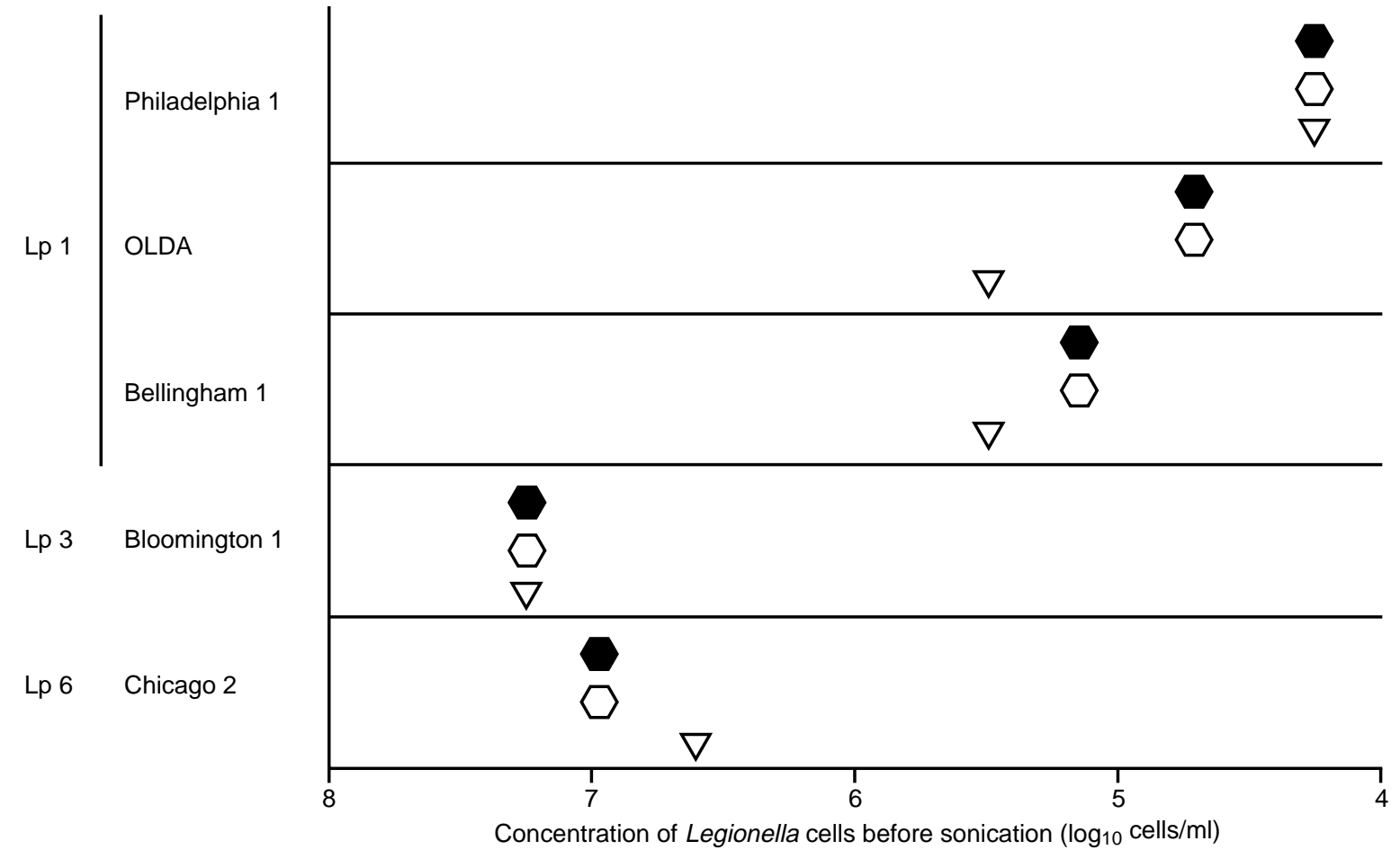

Fig. 1. Lower detection limits of sonicated L. pneumophila (Lp) cells with BinaxNOW Biotest EIA $(\nabla)$.

the Binax and the Biotest EIA were 93.7\% and 92.6\%, respectively.

The majority of the isolates obtained from these patients belonged to the monoclonal subgroups that were recognised by MAb 3/1 [12]. This antibody has the same specificity as MAb2 of the international panel [13] and recognises the subgroups Philadelphia, Allentown, Benidorm, France and Knoxville. Only 2 (2.8\%) of 72 urine samples from such patients were negative by either the BinaxNOW or the Binax EIA and only 3 $(4.2 \%)$ of 72 by the Biotest EIA, whereas the negative rates for the Bellingham group (eight patients) were $38 \%$ for both the BinaxNOW and Binax EIA and 50\% for the Biotest EIA (Table 1). For each assay, the positive rates were significantly less $(p<0.001)$ for the Bellingham group than for the group of MAb 3/1positive isolates. Subgroup OLDA was not included in the calculation because only three cases were identified.

L. pneumophila strains not belonging to serogroup 1 were isolated from 22 patients (Table 2). Only three specimens (14\%) were positive with either BinaxNOW (isolates belonged to serogroups 3, 6 or could not be typed) and Binax EIA (isolates belonged to serogroups 3,5 or could not be typed). The sensitivity of the Biotest EIA (10 of 22 samples) was significantly greater $(p<0.012)$.

The urine samples from culture-proven cases were collected from day 1 to day 33 after onset of symptoms, most of them (90\%) up to day 15 . Differences in the sensitivity of the tests in relation to the collection time were not found.

\section{Detection of Legionella antigens in urine samples from patients diagnosed by seroconversion}

Samples from 70 patients with Legionella infections confirmed by seroconversion to $L$. pneumophila serogroup 1 (group C) were included in this study. The sensitivities of the BinaxNOW assay and the Binax EIA were 78.6 and $80.0 \%$, respectively. The sensitivity

Table 1. Detection of antigenuria in L. pneumophila serogroup 1 cases by three urinary antigen assays

\begin{tabular}{lcccc}
\hline Monoclonal subgroup & & & \\
of the isolated \\
$\begin{array}{l}\text { L. pneumophila } \\
\text { serogroup 1 strain }\end{array}$ & $\begin{array}{c}\text { Number of } \\
\text { specimens tested }\end{array}$ & BinaxNOW & Binax EIA & Biotest EIA \\
\cline { 3 - 5 } MAb 3/1 positive* & 73 & $72(98.6)$ & $71(97.3)$ & $71(97.3)$ \\
OLDA & 3 & $3(100)$ & $3(100)$ & $2(66.7)$ \\
Bellingham & 8 & $5(62.5)$ & $5(62.5)$ & $4(50.0)$ \\
Not known & 11 & $11(100)$ & $10(90.9)$ & $11(100)$ \\
Total & 95 & $91(95.8)$ & $89(93.7)$ & $88(92.6)$ \\
\hline
\end{tabular}

*Monoclonal subgroups Philadelphia, Allentown, Benidorm, France and Knoxville. 
Table 2. Comparison of sensitivities of three commercially available Legionella urinary antigen assays

\begin{tabular}{|c|c|c|c|c|c|}
\hline \multirow{2}{*}{$\begin{array}{l}\text { Patient } \\
\text { group }\end{array}$} & \multirow[b]{2}{*}{ Legionellosis confirmed by } & \multirow{2}{*}{$\begin{array}{l}\text { Number of } \\
\text { specimens } \\
\text { tested }\end{array}$} & \multicolumn{3}{|c|}{ Number $(\%)$ of positive specimens } \\
\hline & & & BinaxNOW & Binax EIA & Biotest EIA \\
\hline $\mathrm{A}+\mathrm{B}+\mathrm{C}$ & $\begin{array}{l}\text { Isolation of L. pneumophila or seroconversion to } \\
\text { L. pneumophila serogroup } 1 \text { or both }\end{array}$ & 187 & $149(79.7)$ & $148(79.1)$ & $156(83.4)$ \\
\hline $\mathrm{A}+\mathrm{B}$ & Isolation of L. pneumophila & 117 & $94(80.3)$ & $92(78.6)$ & $98(83.8)$ \\
\hline A & Isolation of L. pneumophila serogroup 1 & 95 & $91(95.8)$ & $89(93.7)$ & $88(92.6)$ \\
\hline B & Isolation of $L$. pneumophila non-serogroup 1 & 22 & $3(13.6)$ & $3(13.6)$ & $10(45.4)$ \\
\hline $\mathrm{C}$ & Seroconversion to $L$. pneumophila serogroup 1 & 70 & $55(78.6)$ & $56(80.0)$ & $58(82.9)$ \\
\hline $\mathrm{D}$ & $\begin{array}{l}\text { Urinary antigen detection with Binax EIA or Biotest } \\
\text { EIA (or both) only }\end{array}$ & 69 & $63(91.3)$ & $66(95.7)$ & $67(97.1)$ \\
\hline
\end{tabular}

See Materials and methods for definitions of groups A-D.

of the Biotest EIA was only slightly higher at $82.6 \%$ (Table 2).

\section{Detection of antigenuria by BinaxNOW in urine samples that were positive by Binax or Biotest EIA, or both}

To further test the utility of the BinaxNOW assay, 69 urine samples already shown to be positive by the Binax or the Biotest EIA, or both (group D) were also tested (Table 2). For these patients, culture for legionellae and serology for seroconversion gave negative results or were not done. Sixty-one samples $(88.4 \%)$ were positive in all three assays. Four samples were negative only by BinaxNOW and three samples were positive only by either Binax or Biotest EIA. In one case both BinaxNOW and Biotest yielded positive results, whereas the Binax EIA was negative. In general, different results for single assays were obtained only in cases of weak reactivity, showing that the individual cut-off values play a role.

\section{Sensitivity of BinaxNOW in comparison to Binax and Biotest EIA}

Samples from 187 cases of Legionnaires' diseases confirmed by isolation of L. pneumophila or seroconversion to $L$. pneumophila serogroup 1 , or both, were analysed. The overall sensitivities of the BinaxNOW and the Binax were approximately the same $(79.7 \%$ and $79.1 \%$, respectively). The Biotest EIA detected $83.4 \%$ (Table 2). Eleven (5.9\%) of the 187 cases $(5.9 \%)$ confirmed by culture or seroconversion were positive in the BinaxNOW assay but negative by Binax or Biotest EIA, or both.

\section{Discussion}

Shortly after the historic outbreak of Legionnaires' disease in Philadelphia in 1976 [14], Tilton [15] and Berdal et al. [16] developed immunoassays for detection of soluble antigens in urine specimens. Because this diagnostic tool has many advantages compared with other methods, efforts to develop specific and sensitive assays are still ongoing. The most recent development is the BinaxNOW Legionella
Antigen assay based on immunochromatography, which makes it possible to detect antigenuria within a very short time and requires no specialised laboratory equipment.

As the results of the BinaxNOW assay are read visually, the end-point is subjective and consequently it is not so easy to calculate the specificity and sensitivity accurately. In some cases the present study found very weak bands, which were only just visible by eye after the recommended reaction time of $15 \mathrm{~min}$ but could be read with confidence as 'positive' $45 \mathrm{~min}$ later. This phenomenon was seen only in samples from confirmed cases, which, as judged by the low ODs in the Binax or Biotest EIAs, had very low concentrations of antigens (sometimes below the cut-off point). In contrast, the same phenomenon was not observed when testing urine specimens from patients with pneumonia caused by other agents or from urinary tract infections, although eight of these samples also showed very weak bands after $15 \mathrm{~min}$. Heat treatment before testing had no influence on these observations. According to the manufacturer's guidelines, these samples should be considered positive, hence the specificity calculated in this study would be $97.1 \%$. Based on the findings of the present study, it is recommended that 'very slow positive' results can only be judged to be positive with any confidence if the intensity of the band has increased 45 min later. Nevertheless, the final decision is not easy, especially for workers who have only little experience with the test. Therefore, similarly to an 'equivocal' result in an EIA, it is suggested that such results should be interpreted cautiously and should be examined by two workers or examined in a second assay, or both.

Following the modifications described above, the specificity of the BinaxNOW was estimated to be $100 \%$ for patients with pneumonia of other aetiologies and for samples from urinary tract infections. These latter samples mostly contained gram-negative bacteria, among which were 29 containing Pseudomonas spp. which have been reported to cross-react with antisera to Legionella [17]. These data emphasise the high specificity of Legionella urinary antigen assays that was found with the Binax and Biotest EIA $[9,18]$. 
Published sensitivity data for Legionella urinary antigen assays have so far related only to cultureproven cases in general and have not discriminated between serogroups or MAb subgroups [2, 6, 18, 19]. This is largely due to the fact that infections caused by L. pneumophila serogroups 2-15 and some MAb subgroups of serogroup 1 occur only very rarely $[12,20-22]$. Therefore, the present study included the examination of standardised antigen preparations of known bacterial cell concentration for a range of serogroups and subgroups. The values given by concentrations are unlikely to be directly comparable with soluble antigen concentrations in the urine samples, because under in-vivo conditions legionellae are able to shed lipopolysaccharides (LPS) from the cell wall [23]. Nevertheless, the differences seen between the lower detection limits obtained for the antigen preparations derived from the various strains correlated well with the diagnostic probability of detecting antigenuria in patients infected by these different subgroups and serogroups. As shown in Fig. 1 , all three assays had the highest sensitivity for $L$. pneumophila serogroup 1 strain Philadelphia. To obtain a positive result for strains OLDA or Bellingham by Biotest EIA, >10-fold higher concentrations were necessary. Compared with the Biotest EIA, these differences were not so extreme for the two Binax assays. For the other serogroups examined, antigen concentrations had to be $>500$-fold higher to obtain positive results. Dominguez et al. [18] reported that both the Binax and Biotest EIA were able to detect soluble antigens from all serogroups of $L$. pneumophila, but they did not quantify the amounts of antigens necessary for recognition of the individual serogroups. Heating of antigen preparations $\left(95^{\circ} \mathrm{C}, 10 \mathrm{~min}\right)$ did not lead to a decrease in sensitivity, indicating that the heat-stable LPS is the major immunogenic component which is recognised by the three kits used.

Seventy-three of the 117 culture-proven infections were caused by strains identified as serogroup 1 monoclonal subgroups Philadelphia, Allentown, Benidorm, France or Knoxville. All strains belonging to these subgroups are characterised by having a common LPS epitope which was recognised by MAbs 3/1 [12] and MAb 2 [13]. This epitope is associated with the 8-O- acetyl group of the legionaminic acid, being the monomer of the O-chain of the LPS, [12] and this component is highly immunogenic. All the three antigen assays used were able to detect antigenuria in $>97 \%$ of cases confirmed by isolation of MAb 3/1-positive isolates (Table 1). Only eight isolates were identified as subgroup Bellingham. The detection of antigenuria in this group was significantly less than with MAb 3/1positive isolates. As discussed above, this reflects the different detection limits found for simulated antigens of the MAb 3/1-positive strain Philadelphia and the monoclonal subgroup Bellingham (Fig. 1). The sensitivities of the three antigen assays for infections caused by strains of the monoclonal subgroup OLDA could not be evaluated because only three urine samples were available from patients infected by such strains.

The present study tested 22 urine samples from patients with legionellosis caused by non-serogroup 1 strains. Both the BinaxNOW ICT assay and Binax EIA are marketed by the manufacturer as kits for detection of L. pneumophila serogroup 1 urinary antigen. Nevertheless, in three $(14 \%)$ of the 22 cases it was possible to detect antigenuria. Benson et al. [24] obtained positive urinary antigen results by Binax EIA for three serogroup 6 and two serogroup 8 cases. In the present study the sensitivity was significantly higher with the Biotest EIA, which is marketed as recognising all serogroups of L. pneumophila $(46 \%, \mathrm{p}<0.012)$. However, compared with serogroup 1 cases the sensitivity of this EIA for non-serogroup $1 \mathrm{~L}$. pneumophila was very low $(p<0.001)$.

Compared with culture-proven cases caused by $L$. pneumophila serogroup 1, patients with seroconversion to L. pneumophila serogroup 1 had a lower rate of antigenuria as detected by all three assays used $(p \leqslant 0.056)$. This could be due partly to the fact that patients with legionellosis caused by serogroups other than serogroup 1 can also develop high levels of antibodies against common antigens of this species. However, samples from three patients with seroconversion to only serogroups 3,5 , or 4 and 5 were also examined (data not shown). None of these was positive in any of the three assays, again indicating the low sensitivity of urinary antigen detection kits for cases not caused by serogroup 1 .

Of the 187 Legionella infections confirmed by culture or seroconversion, or both, $163(87.2 \%)$ were positive by at least one urinary antigen assay, but only $74.3 \%$ were positive in all of them. The combination of being positive in one assay and negative in another is typical for specimens containing low concentrations of Legionella antigens or containing antigens of serogroups other than serogroup 1. For eight culture-proven or serologically proven cases that were positive by BinaxNOW, confirmation by the other two assays was successful in only one kit. Furthermore, three cultureproven cases (L. pneumophila serogroups 1, 3 and 6) were positive only by BinaxNOW. In such cases, concentration of the urine specimens has been recommended by some workers. Dominguez et al. [19] were able to increase the sensitivity of Binax EIA and Biotest EIA by 25 and $20 \%$, respectively, when the urine samples were concentrated by a factor of 25 . Compared with EIA with concentrated samples as reference, Dominguez et al. [18] increased the sensitivity of BinaxNOW through concentration of samples from 56 to $97 \%$. However, the introduction of a concentration step before testing with BinaxNOW would compromise the very advantages that characterise the ITC, i.e., its rapidity and the fact that it does not need specialised laboratory equipment. 
The present study confirms that the BinaxNOW ICT assay is suitable for specific Legionella urinary antigen detection with at least the same sensitivity for infections caused by L. pneumophila serogroup 1 as the Binax and Biotest EIAs. The results were normally available in $15 \mathrm{~min}$, in the case of strong reactions $<5 \mathrm{~min}$. Furthermore, no specialised laboratory equipment is necessary. However, the present study also demonstrated that all three kits tested often failed to detect antigenuria where infections were due to strains of MAb subgroup Bellingham. Furthermore, infections caused by $L$. pneumophila serogroups $2-15$ are diagnosed only rarely by the two Binax assays tested and although the Biotest EIA is better it still lacks sensitivity for this group of patients. Therefore, it remains an open question as to whether infections caused by strains not belonging to the monoclonal subgroup MAb 3/1-positive (or MAb2-positive [12, 13]) are really so rare as is reported in many studies, or if these infections are significantly underdiagnosed partly because of the low sensitivity of all commercially available urinary tests. Data from Denmark could indicate that infections caused by non-serogroup 1 legionellae are underdiagnosed in some countries. During a period of 5 years (1994-1998), Legionella spp. were isolated from 139 patients; only $78(56 \%)$ of the isolates belonged to serogroup 1, of which approximately $76 \%$ were reactive with the MAb 3/1 (data not published). The diagnosis of such infections has especially far-reaching implications for nosocomially acquired legionellosis, which is caused significantly more often by MAb 3/1(MAb 2)-negative or non-serogroup 1 isolates than community-acquired legionellosis $[26,27])$. Therefore, beside urinary antigen detection, DFA and attempts to culture legionellae remain important tools for diagnosing legionellosis in this group of patients. However, in the context of community-acquired pneumonia requiring admission to hospital, detection of urinary antigen is undoubtedly the best way to attempt to diagnose Legionella infection.

We thank Dr S. Monecke for typing of the isolates cultured from patients with urinary tract infections and Ines Wolf, Nita Doshi and Ilse Kjaerulff for excellent technical assistance.

\section{References}

1. Heath $\mathrm{CH}$, Grove DI, Looke DFM. Delay in appropriate therapy of Legionella pneumophila associated with increased mortality. Eur J Clin Microbiol Infect Dis 1996; 15: 286-290.

2. Ruf B, Schürmann D, Horbach I, Fehrenbach FJ, Pohle HD. Prevalence and diagnosis of Legionella pneumonia: a 3-year prospective study with emphasis on application of urinary antigen detection. J Infect Dis 1990; 162: 1341-1348.

3. Edelstein PH, Meyer RD. Legionella. Adv Pathol Lab Med 1994; 8: $149-167$

4. Edelstein PH. The laboratory diagnosis of Legionnaires' disease. Semin Respir Infect 1987; 2: 235-241.

5. Harrison TG, Taylor AG. Demonstration of legionellae in clinical specimens. In: Harrison TG, Taylor AG (eds) A laboratory manual for Legionella. Chichester, John Wiley and Sons. 1988: 103-112.
6. Aguero-Rosenfeld ME, Edelstein PH. Retrospective evaluation of the Du Pont radioimmunoassay kit for detection of Legionella pneumophila serogroup 1 antigenuria in humans. J Clin Microbiol 1988; 26: 1775-1778.

7. Kohler RB, Zimmerman SE, Wilson E et al. Rapid radioimmunoassay diagnosis of Legionnaires' disease: detection and partial characterization of urinary antigen. Ann Intern Med 1981; 94: 601-605.

8. Kohler RB, Allen SD, Wheat LJ, White AC. Development and evaluation of diagnostic radiometric assays for serogroup $4 \mathrm{~L}$. pneumophila urinary antigens. Diagn Microbiol Infect Dis 1987; 6: 101-107.

9. Plouffe JF, File TM, Breiman RF et al. Reevaluation of the definition of Legionnaires' disease: use of the urinary antigen assay. Clin Infect Dis 1995; 20: 1286-1291.

10. Harrison TG, Taylor AG. The diagnosis of Legionnaires' disease by estimation of antibody levels. In: Harrison TG, Taylor AG (eds) A laboratory manual for Legionella. Chichester, John Wiley and Sons. 1988: 113-135.

11. Helbig JH, Kurtz JB, Castellani Pastoris M, Pelaz C, Lück PC. Antigenic lipopolysaccharide components of Legionella pneumophila recognized by monoclonal antibodies: possibilities and limitations for division of the species into serogroups. $J$ Clin Microbiol 1997; 35: 2841-2845.

12. Helbig JH, Lück PC, Knirel YA, Witzleb W, Zähringer U. Molecular characterization of a virulence-associated epitope on the lipopolysaccharide of Legionella pneumophila serogroup 1. Epidemiol Infect 1995; 115: 71-78.

13. Joly JR, McKinney RM, Tobin OJ, Bibb WF, Watkins ID, Ramsay D. Development of a standardized subgrouping scheme for Legionella pneumophila serogroup 1 using monoclonal antibodies. J Clin Microbiol 1986; 23: 768-771.

14. Fraser DW, Tsai TR, Orenstein W et al. Legionnaires' disease: description of an epidemic of pneumonia. N Engl J Med 1977; 297: 1189-1197.

15. Tilton RC. Legionnaires' disease antigen detected by enzymelinked immunosorbent assay. Ann Intern Med 1979; 90: 697-698.

16. Berdal BP, Farshy CE, Feeley JC. Detection of Legionella pneumophila antigen in urine by enzyme-linked immunospecific assay. J Clin Microbiol 1979; 9: 575-578.

17. Tenover FC, Edelstein PH, Goldstein LC, Sturge JC, Plorde JJ. Comparison of cross-staining reactions by Pseudomonas spp. and fluorescein-labeled polyclonal and monoclonal antibodies directed against Legionella pneumophila. J Clin Microbiol 1986; 23: 647-649.

18. Dominguez JA, Gali N, Pedroso P et al. Comparison of the Binax Legionella Urinary Antigen Enzyme Immunoassay (EIA) with the Biotest Legionella Urin antigen EIA for detection of Legionella antigen in both concentrated and nonconcentrated urine samples. J Clin Microbiol 1998; 36: 2718-2722.

19. Dominguez J, Gali N, Matas L et al. Evaluation of a rapid immunochromatographic assay for the detection of Legionella antigen in urine samples. Eur $J$ Clin Microbiol Infect Dis 1999; 18: 896-898.

20. Dournon E, Bibb WF, Rjagopalan P, Desplaces N, McKinney RM. Monoclonal antibody reactivity as a virulence marker for Legionella pneumophila serogroup 1 strains. J Infect Dis 1988; 157: 496-501.

21. Reingold AL, Thomason BM, Brake BJ, Thacker L, Wilkinson HW, Kuritsky JN. Legionella pneumonia in the United States: the distribution of serogroups and species causing human illness. J Infect Dis 1984; 149: 819.

22. Stout JE, Joly J, Para $\mathrm{M}$ et al. Comparison of molecular methods for subtyping patients and epidemiologically linked environmental isolates of Legionella pneumophila. J Infect Dis 1988; 157: 486-495.

23. Susa M, Helbig JH, Marre R. Shedding of the Legionella pneumophila lipopolysaccharide during intracellular multiplication to vesicles of the host cells. In: Abstracts of the 99th General Meeting of the American Society for Microbiology, 1999, Chicago, IL, USA. Abstract D/B-77.

24. Benson RF, Tang PW, Fields BS. Detection of Legionnaires' disease due to multiple serogroups and species of Legionella with the Binax RIA Urinary Antigen Kit and validation of a broad-spectrum ELISA. In: Abstracts of the 37th Interscience Conference on Antimicrobial Agents and Chemotherapy, 1997, Toronto, Ont., Canada. Abstract D-156. 
25. World Health Organization. Epidemiology, prevention, and control of legionellosis. Memorandum from a WHO meeting. Bull World Health Organ 1990; 68: 155-164.

26. Joseph CA, Watson JM, Harrison TG, Bartlett CLR. Nosocomial Legionnaires' disease in England and Wales, 1980-92.
Epidemiol Infect 1994; 112: 329-345.

27. Köhler JR, Maiwald M, Lück PC, Helbig JH, Hingst V, Sonntag H-G. Detecting legionellosis by unselected culture of respiratory tract secretions and developing links to hospital water strains. J Hosp Infect 1999; 41: 301-311. 